\title{
Calcium Binding Protein-Like Immunoreactivity Labels the Terminal Field of Nucleus Laminaris of the Barn Owl
}

\author{
Terry T. Takahashi, ${ }^{1}$ Catherine E. Carr, ${ }^{1}$ Nicholas Brecha, ${ }^{2}$ and Masakazu Konishi ${ }^{1}$ \\ 'Division of Biology, California Institute of Technology, Pasadena, California 91125, and 'Department of Medicine and \\ Brain Research Institute, School of Medicine, University of California, Los Angeles, Los Angeles, California 90024, and \\ Veterans' Administration Medical Center-Wadsworth, Los Angeles, California 90073
}

\begin{abstract}
Nucleus laminaris (NL) is the site at which the timing of sounds arriving in the 2 ears is compared in the auditory system of the barn owl. Earlier studies have reported vitamin D-dependent calcium binding protein (CaBP)-like immunoreactivity in the somata of NL. We report here that CaBPlike immunoreactivity stains the terminal field of NL. The specific CaBP immunoreactivity is localized to a dense plexus of fibers that have bouton-like swellings, usually around unstained somata. This type of immunoreactivity is found in a restricted portion of the central nucleus of the inferior colliculus (ICC), in the anterior division of the ventral lateral lemniscal complex (VLVA), and in the superior olivary nucleus (SO), all of which have been shown by anterograde transport of ${ }^{3} \mathrm{H}$-proline to be innervated by $\mathrm{NL}$. The immunoreactivity is absent from the posterior division of ventral lateral lemniscal complex and from the region that surrounds the portion of ICc innervated by NL. A restricted lesion in NL results in a localized deficit in immunoreactivity in those regions of ICC and VLVA that are known to be innervated by the lesioned area of NL. In adjacent sections processed by the Fink-Heimer method, degenerating axons are present in the region of the deficit in immunoreactivity. ${ }^{3}$
\end{abstract}

To the barn owl (Tyto alba) the interaural differences in the timing and intensity of sounds signify, respectively, the horizontal and vertical coordinates of the source (Knudsen and Konishi, 1979). These 2 cues for sound localization are processed in separate neural pathways (Moiseff and Konishi, 1983; Sullivan and Konishi, 1984; Takahashi et al., 1984). The pathway for processing intensity information begins at nucleus angularis (NA of Fig. 1), one of the two cochlear nuclei, the neurons of which are sensitive to the sound-pressure level in the ipsilateral ear (Sullivan and Konishi, 1984). NA projects bilaterally to the superior olivary nucleus $(\mathrm{SO})$ and contralaterally to certain areas of the lateral lemniscal nuclear group (VLVP of Fig. 1) and the central nucleus of the inferior colliculus (ICc) (Leibler, 1975; Takahashi and Konishi, 1985, and unpublished observations;

\footnotetext{
Received Aug. 26, 1986; revised Dec. 30, 1986; accepted Jan. 27, 1987.

We thank Dr. D.E.M. Lawson for giving us the primary antiserum; Ms. Marianne Cilluflu, Ms. Valerie Leathers, and Dr. A. W. Norman for their assistance in the isolation of calcium binding protein; Dr. Ngozi Erondu for his help in biochemical analysis; and Drs. David C. Van Essen and Leonard Maler for helpful discussions. This study was supported by National Science Foundation Grant BNS8411458 and NIH Grant NS14617 to M. K. and by an A.F.P. Sloan Fellowship and NIH Grant NEI04067 to N.B.

Correspondence should be addressed to Terry Takahashi, Ph.D., Division of Biology 216-76, California Institute of Technology, Pasadena, CA 91125.

${ }^{3}$ See Appendix for list of abbreviations.

Copyright (C) 1987 Society for Neuroscience $0270-6474 / 87 / 061843-14 \$ 02.00 / 0$
}

Conlee and Parks, 1986) that contain neurons sensitive to the difference in sound intensity in the 2 ears (Moiseff and Konishi, 1983; Takahashi and Konishi, 1983).

The pathway that processes timing information originates in nucleus magnocellularis (NM), the other cochlear nucleus, the neurons of which preserve the temporal characteristics of the sound in the ipsilateral ear (Sullivan and Konishi, 1984). In the owl, NM projects exclusively and bilaterally to nucleus laminaris (NL) (Takahashi and Konishi, 1985, and unpublished observations), the avian analog of the medial superior olive. Recent neurophysiological evidence suggests that NL is the site at which the interaural phase differences of spectral components of sounds are encoded (Sullivan and Konishi, 1986). NL, in turn, projects contralaterally to regions of the lateral lemniscal nuclear complex (VLVA) and ICc (Leibler, 1975; Takahashi and Konishi, 1985, and unpublished observations; Conlee and Parks, 1986) that are composed of neurons selective for interaural time differences (Moiseff and Konishi, 1983; Takahashi and Konishi, 1983; Wagner et al., 1986). There is also a sparse projection to the ipsilateral SO (Takahashi and Konishi, 1985, and unpublished observations). Time and intensity pathways finally converge in the external nucleus of the inferior colliculus (ICx), the cells of which are selective for both interaural time and intensity differences (Moiseff and Konishi, 1981, 1983).

Recent immunohistochemical studies have reported the existence of vitamin D-dependent calcium binding protein- $(28,000$ $\mathrm{Da}$ ) like immunoreactivity in the neurons of NM and NL (Jande et al., 1981; Carr et al., 1985). We demonstrate here that a polyclonal antibody raised against this protein (Jande et al., 1981) stains the terminal field of NL and is therefore useful for the delineation of a major part of the time pathway. These results have been presented as an abstract at the annual meeting of the Society for Neuroscience (Takahashi et al., 1986).

\section{Materials and Methods}

Data are based on results from 8 adult barn owls (Tyto alba) of both sexes. Details regarding stereotaxis, surgery, acoustic stimulation, and postoperative care are available elsewhere (Takahashi and Konishi, 1986). Below, we shall refer to the antigen, chick intestinal vitamin D-dependent calcium binding protein $(28,000 \mathrm{Da})$, as "CaBP," and to CaBPlike immunoreactivity as "CaBP immunoreactivity." We use the nomenclature of Karten and Hodos (1967) as modified by Leibler (1975), and of Knudsen (1983).

Immunohistochemistry. Owls used for CaBP immunohistochemistry were given an overdose of pentobarbital (Nembutal, $50 \mathrm{mg} / \mathrm{ml}$; Abbott Laboratories), transcardially exsanguinated with $0.9 \%$ saline (unbuffered), and fixed by perfusion, in all but one case, with a mixture of $4.0 \%$ paraformaldehyde, $1.37 \%$ DL-lysine, and $0.21 \%$ sodium periodate in 0.1 M phosphate buffer ( $\mathrm{pH} 7.40$ ) (sodium periodate-lysine paraformalde- 


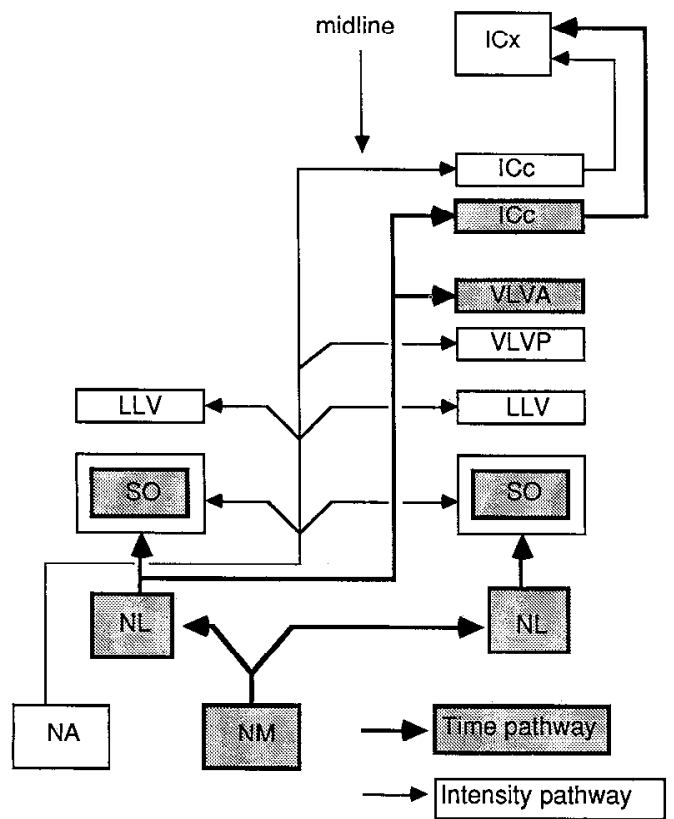

Figure 1. Summary of auditory projections in the brain stem of the barn owl. Shaded boxes represent nuclei of the "time pathway," which originates at nucleus magnocellularis $(N M)$. Empty boxes represent nuclei of the "intensity pathway," which originates at nucleus angularis $(N A)$. The superior olive $(S O)$ is innervated by both nucleus angularis and nucleus laminaris $(N L)$ and is therefore enclosed in both shaded and empty boxes.

hyde). In the one exception, the fixative was $10 \%$ unbuffered formolsaline. Brains were stereotaxically blocked, removed, stored for 4-8 hr in fixative containing sucrose (30\%), and cut frozen on a sliding microtome at $30 \mu \mathrm{m}$ in the coronal plane. Sections were collected and stored in ice-cold $0.1 \mathrm{M}$ PBS in all but the one case perfused with $10 \%$ formolsaline. In the latter, sections were collected and stored (for 1 month) in the fixative, but were washed at room temperature for $24 \mathrm{hr}$ in PBS prior to processing for immunohistochemistry.

Sections were incubated for $24-48 \mathrm{hr}$ at $4^{\circ} \mathrm{C}$ in primary antiserum diluted to $1: 10,000$ (in PBS containing $0.5 \%$ Triton) in all cases except for the serial dilution controls (below). Sections were subsequently treated according to the peroxidase-antiperoxidase or avidin-biotin (Vector Laboratories) method in conjunction with horseradish peroxidase/diaminobenzidine histochemistry. The quality of the immunoreactivity was comparable whether peroxidase-antiperoxidase or avidin-biotin protocols were followed and whether the tissue was fixed with sodium periodate-lysine paraformaldehyde and stored in PBS or fixed and stored in formol-saline.

Controls. The antiserum that we used was kindly provided by Dr. D. Eric M. Lawson of the University of Cambridge and has been reported to be monospecific for chick intestinal CaBP protein (CaBP, 28,000 Da) in the brain of the chick and rat (Jande et al., 1981) and of Eigenmannia, a weakly electric fish (Maler et al., 1984). Absorption controls (Pickel, 1981) were also run in the owl. Primary antiserum was first diluted to $1: 10,000$ in PBS (with $0.5 \%$ Triton) containing an excess quantity $(0.5 \times$ $\left.10^{-5} \mathrm{M}\right)$ of CaBP purified from the duodenum of chicks according to the protocol of Friedlander and Norman (1977). The antigen-antiserum mixture (absorbed primary antiserum) was stored for $12 \mathrm{hr}$ at $4^{\circ} \mathrm{C}$ before sections were incubated therein. Sections were incubated for $48 \mathrm{hr}$ at $4^{\circ} \mathrm{C}$ in absorbed antiserum or in ordinary unabsorbed antiserum. All sections were simultaneously processed by the avidin-biotin protocol. Antibodies specific for CaBP bind to the purified antigen and are thus unavailable for binding to the antigen present in situ. CaBP immunoreactivity, therefore, would not be observed in sections incubated in absorbed primary antiserum.

For serial dilution controls (Pickel, 1981), sections were incubated for $36 \mathrm{hr}\left(\right.$ at $\left.4^{\circ} \mathrm{C}\right)$ in primary antiserum diluted to $1: 2500,1: 10,000$, $1: 20,000,1: 30,000,1: 50,000,1: 100,000$, and 1:300,000. Sections were then treated by the avidin-biotin method. Except for the primary antiserum, all sections shared common reaction baths and were incubated simultaneously. We also quantified the results of the serial dilutions by exploiting the spot-metering capability of the photographic system (Nikon UFX-II; Nippon Kogaku) attached to our light microscope (Nikon Optiphot). Specifically, we mcasurcd the amount of time required for a constant level of exposure of photographic film having a given sensitivity (we chose ASA 12) at different spots within an immunoreactive zone in the ICc. The metering spot is designed to cover $1 \%$ of the area in view at any given magnification (we used $100 \times$ total magnification). Sections were moved with respect to the spot so that exposure times were measured at all parts within the zone in the inferior colliculus (about $4 \mathrm{~mm}^{2}$ ). Light intensity and exposure time are reciprocally related. Thus, the reciprocal of the exposure time taken at each spot was evaluated and the values averaged as an index of the light transmitted through the section (i.e., the "lightness" of a section). The reciprocal of the exposure time was plotted against the reciprocal of primary antiserum dilution.

Autoradiography. The autoradiographic results presented below will be included in another report (T. Takahashi and M. Konishi, unpublished observations). L-2,3-3 $\mathrm{H}$-proline ( $40 \mathrm{Ci} / \mathrm{mmol}$; New England $\mathrm{Nu}$ clear) was dried and reconstituted with $0.9 \%$ saline to a concentration of $50 \mu \mathrm{Ci} / \mu \mathrm{l}$ and loaded into a glass micropipette having an inner diameter of $20 \mu \mathrm{m}$ at the tip. Owls were anesthetized by intramuscular injections of ketamine hydrochloride $\left(0.1 \mathrm{~cm}^{3} / \mathrm{hr}\right.$; Vetalar, $100 \mathrm{mg} / \mathrm{ml}$; Bristol Laboratories) and were kept warm with a heating pad throughout the injection procedure. The micropipette was targeted stereotaxically and lowered into NL with a remote-controlled, stepping motor-coupled microdrive. Evoked potentials or "neurophonic" responses (see Sullivan and Konishi, 1986) that can be recorded in NL in response to diotic stimulation are sharply tuned in frequency. The frequency that evoked the strongest neurophonic response (best frequency) was noted for all injection sites. ${ }^{3} \mathrm{H}$-proline was iontophoresed from the micropipette with a $+2 \mu \mathrm{A}$ pulsed DC current ( $5 \mathrm{sec}$ on, $5 \mathrm{scc}$ off) applicd for 15-20 min. After a 2-3 d survival period, the owls were killed with an overdose of pentobarbital, exsanguinated with saline, and fixed with formolsaline. Brains were cut in the coronal plane at $30 \mu \mathrm{m}$ and processed for autoradiography according to the protocol of Cowan et al. (1972). We used Kodak NTB-2 emulsion.

Lesion experiments. We tested the effects of lesioning NL on the immunoreactivity in the auditory nuclei of the owl's brain stem in 2 cases. Owls were anesthetized for lesioning experiments with ketamine hydrochloride. NL was lesioned electrolytically by passage of $+0.2 \mathrm{~mA}$ of DC current for $30 \mathrm{sec}$ or $+2 \mu \mathrm{A}$ (DC) for $15 \mathrm{~min}$. After a $10 \mathrm{~d}$ survival period, the owls were given an overdose of pentobarbital, exsanguinated with saline, and fixed with $10 \%$ formol-saline; the brains were cut at $30 \mu \mathrm{m}$ in the coronal plane. One series of every third section was processed for immunohistochemistry (as above), and alternate scrics werc processed for degeneration (Fink and Heimer, 1967) and Nissl staining.

\section{Results}

CaBP immunoreactivity is observed in many nuclei throughout the brain stem. Individual nuclei are characterized by the differential staining of somata, neuropil, and fibers. Although staining patterns throughout the brain lead us to believe that $\mathrm{CaBP}$ immunoreactivity will be useful in delineating numerous tracts and nuclei of the owl's brain, we shall restrict our analysis and description to a particular type of immunoreactive staining found in the auditory system.

This staining, which is found within a subdivision of the ICc (Fig. 2), in the VLVA (Fig. 3), and, in lower densities, in the SO (Fig. 4), consists of a network of tangled fibers that end in bouton-like swellings, usually around unstained somata. We will refer to this staining pattern hereafter as the "immunoreactive fiber plexus." The staining is notable more for its texture than for the absolute density of its immunoreactivity. The external nucleus of the inferior colliculus (ICx), for example, is strongly immunoreactive, but the staining is diffuse and the fibrous quality is absent. The appearance of the fibers in VLVA and SO is identical to that in ICc, except that the density is lower in SO.

In ICc, the immunoreactive fiber plexus forms a vertical strip in the center of ICc and extends anteriorly from the level, rough- 

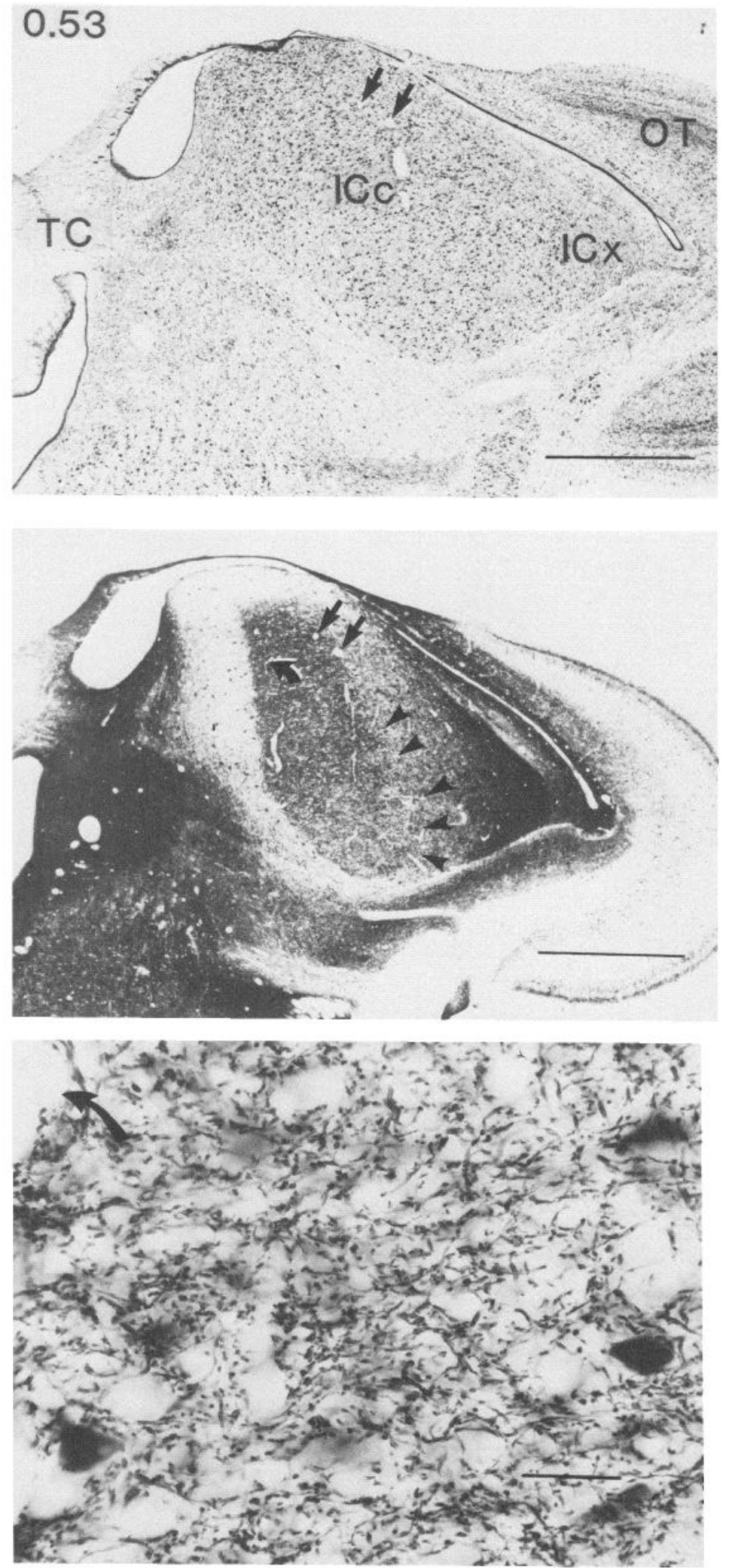

Figure 2. Immunoreactivity in inferior colliculus $(I C)$. Top, Cresyl violetstained coronal section through the midbrain of the right side (dorsal and lateral are up and right, respectively). Number at the upper left is the "normalized anteroposterior (ap) level," i.e. the distance of the section from the posterior pole of the nucleus divided by the length of the ap dimension of the nucleus. Scale bar, $1 \mathrm{~mm}$. Center, Adjacent (to cresyl violet-stained section) section treated for CaBP immunoreactivity. The immunoreactive fiber plexus is seen in the central nucleus $(I C C)$, with arrowheads delimiting its lateral border. The 2 small, straight arrows located dorsolaterally point to fiducial blood vessels that are common to the sections depicted in the top and center photographs. Scale bar, $1 \mathrm{~mm}$. Bottom, High-magnification photo of the immunoreactive fiber plexus taken from the dorsomedial region of the section shown in center photo. At this magnification, fine fibers and swellings are resolvable. The curved arrow at the upper left points to the blood vessel that is also visible in the lower-power photo (center photo, curved black on white arrow). Scale bar, $40 \mu \mathrm{m}$. ly, at which the tectal commissure (TC) becomes confluent across the midline (about 0.4 of the length of the ICc from its caudal pole). Thus, it occupies only a portion of ICc. The border of the immunoreactive fiber plexus is readily discernible although the adjacent neuropil is diffusely stained and contains darkly stained somata.

The immunoreactive fiber plexus is confined to VLVA (Fig. 3 ), although stained axons course through the posterior subdi- 


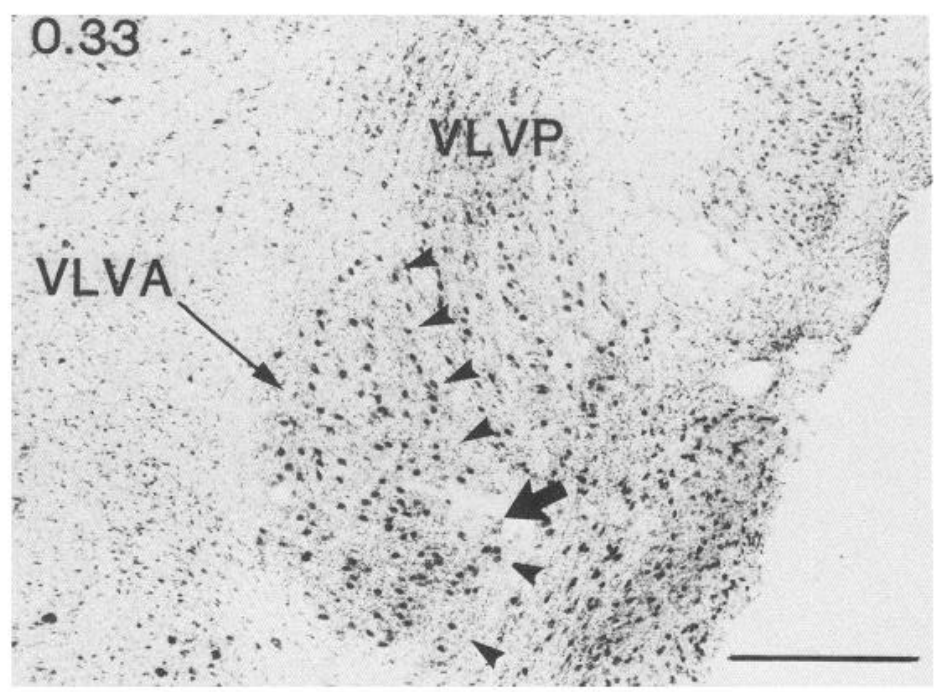

Figure 3. Immunoreactivity in nucleus ventralis lemnisci lateralis $(V L V)$. Top, Cresyl violet-stained section through the anterior $(V L V A)$ and posterior $(V L V P)$ subdivisions of the VLV complex of the right side. Arrowhead tips demarcate the boundary between the 2 subdivisions. Scale bar, $0.5 \mathrm{~mm}$. Center, Adjacent section treated for $\mathrm{CaBP}$ immunoreactivity. The immunoreactive fiber plexus is confined to VLVA (to the left of arrowhead tips), although CaBP-positive fascicles course through VLVP. The heavy, straight arrow indicates a blood vessel common to the top and center sections. Scale bar, $0.5 \mathrm{~mm}$. Bottom, High-magnification photo of the immunoreactive fiber plexus in VLVA taken from the part of VLVA that includes the blood vessel (curved arrow at top), indicated by the curved arrow in the center photo. Scale bar, $40 \mu \mathrm{m}$.
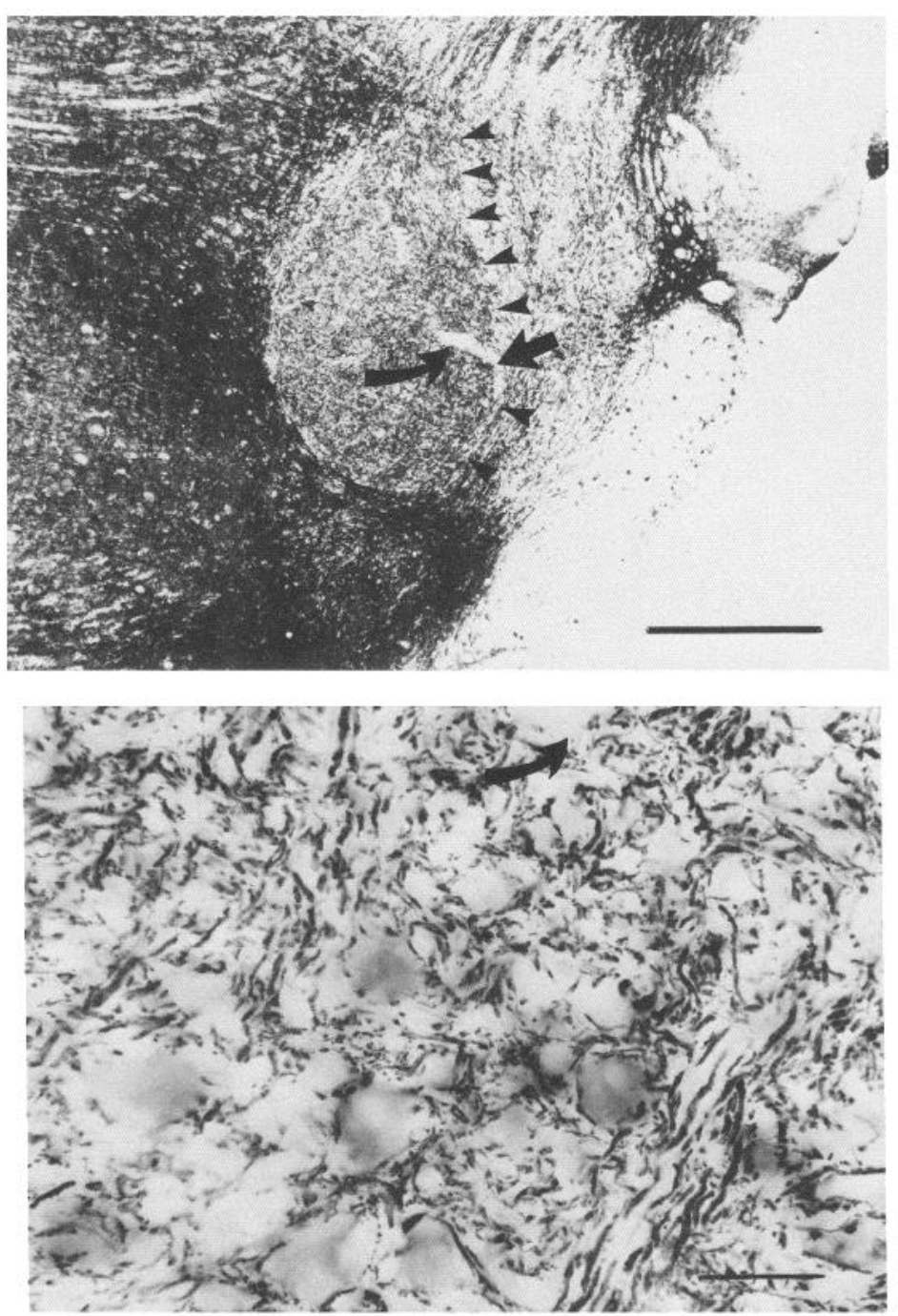

vision (VLVP), the cell group with which VLVA is contiguous laterally. No immunoreactive plexus, however, is observed in VLVP. In SO (Fig. 4), the fiber plexus is sparse everywhere, but is densest in the ventral portion. In addition, fascicles of immunoreactive axons are seen coursing through the nucleus.

\section{Controls}

The staining described above is dependent on the presence of primary antibody and is therefore not an artifact of the methods used to render visible the immunoreactivity (the peroxidase- 

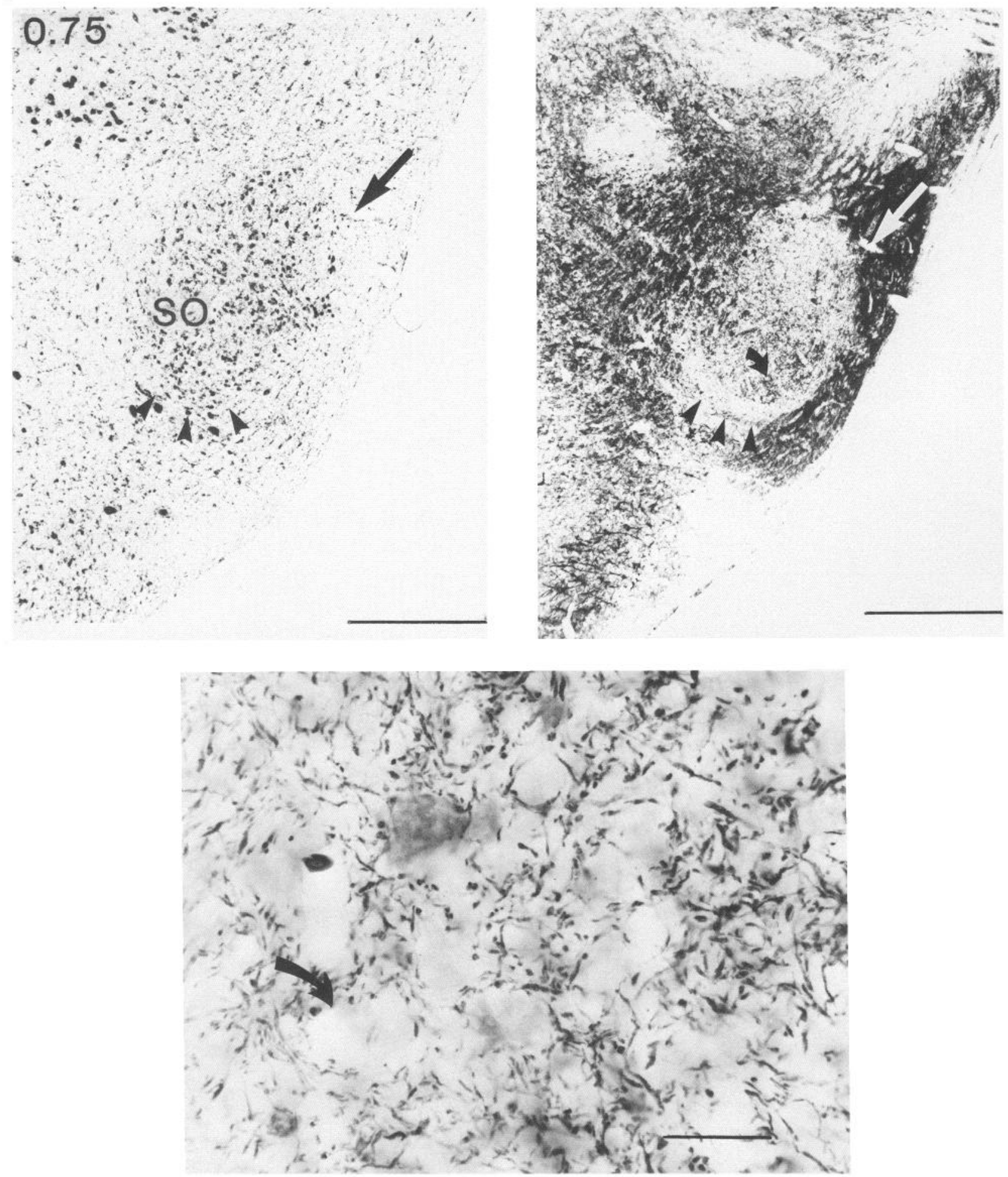

Figure 4. Immunoreactivity in superior olivary nucleus (SO). Top left, Cresyl violet-stained coronal section through right SO. Arrowhead tips indicate the ventral nuclear boundary. Scale bar, $0.5 \mathrm{~mm}$. Top right, Adjacent coronal section treated for CaBP immunoreactivity. The immunoreactive fiber plexus, which is sparse in SO, is concentrated in the ventral aspect of the nucleus. The large white on black arrow points to a blood vessel that also appears on the cresyl violet-stained section to the left (top left, large black arrow). Scale bar, $0.5 \mathrm{~mm}$. Bottom, High-magnification view of the immunoreactive fiber plexus in SO taken from the vicinity of the top right section, indicated by the small curved arrow. The same blood vessel is indicated by a large curved arrow in the bottom photo. Scale bar, $40 \mu \mathrm{m}$.

antiperoxidase or avidin-biotin techniques). As shown in Figure 5 , serial dilution of primary antibody results in a corresponding decrease in staining density. A plot of inverse exposure time as a function of inverse primary antibody dilution, shown in Figure
6, also demonstrates that the sections become darker as primary antibody concentration increases. The plot further suggests that at 1:20,000, maximal staining levels are attained.

In sections incubated in primary antiserum that was absorbed 

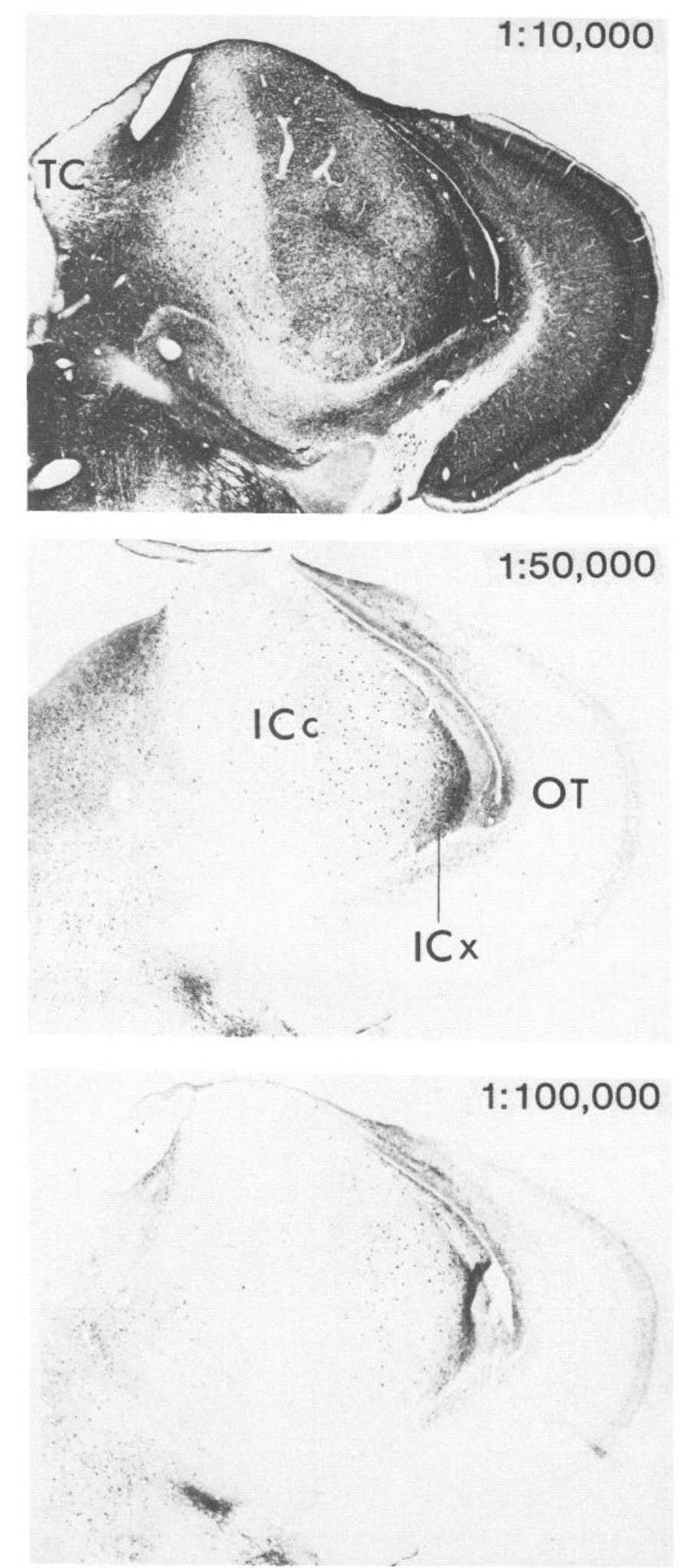

Figure 5. Serial dilution controls. Photomicrographs of sections incubated in primary antiserum diluted to $1: 10,000(t o p)$, to $1: 50,000$ (middle), and to $1: 100,000$ (bottom). All sections were photographed at the same light intensity and exposure time. The section shown at the bottom (1:100,000 dilution), however, was printed at a slightly longer exposure time than were the other 2 sections in order to obtain an appreciable image.

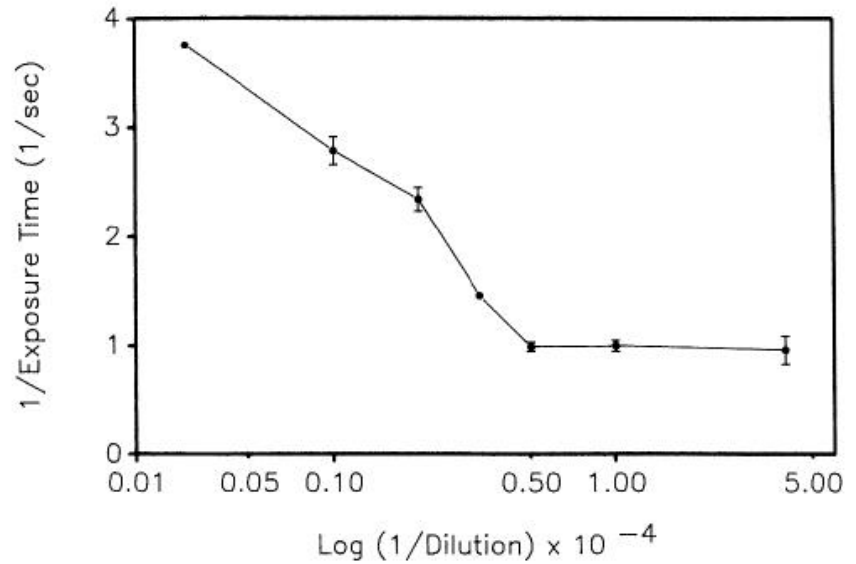

Figure 6. Inverse exposure time as a function of inverse primary antiserum dilution. The reciprocal of exposure time is an index of the amount of light transmitted through the section to the light meter of the photomicroscope. Data points represent the average of 1/exposure time values $( \pm \mathrm{SD})$ obtained across the entire area of the immunoreactive fiber plexus in ICc.

with purified antigen, the immunoreactive fiber plexus is not observed. The immunoreactive fiber plexus is observed in nearby sections that were incubated in normal (not absorbed with antigen) primary antiserum at the same time.

\section{Projections of $N L$}

The projection of NL terminates in areas containing the immunoreactive fiber plexus. The projection of NL is the subject of a future report (T. Takahashi and M. Konishi, unpublished observations) and will be described only briefly here.

Autoradiographic label in the contralateral VLVA typical of ${ }^{3} \mathrm{H}$-proline injections into NL is shown in Figure $7 A$. Injections into other parts of NL label different parts of VLVA, but label never extends beyond the cytoarchitectural boundary of the nucleus as long as injections are confined to NL. The ipsilateral VLVA is never labeled. The label in the ipsilateral SO, as can be seen in Figure $7 B$, consists of a light scattering of grains between heavily labeled fascicles passing through and dorsomedial to the nucleus. Label is never observed over the contralateral SO. Autoradiographic label in the contralateral ICc resulting from 4 injections (Fig. 8, $A-D$ ) along the tonotopic axis (roughly from posterolateral to anteromedial) of NL is shown in Figure 9. Three of the 4 injections were made in one bird, owl 174 (Fig. 8, $A-C$ ), at sites in which the evoked potential or "neurophonic" response (see Sullivan and Konishi, 1986) was tuned to $5.1 \mathrm{kHz}$ (Fig. $8 A$ ), $6.4 \mathrm{kHz}$ (Fig. $8 B$ ), and $7.4 \mathrm{kHz}$ (Fig. $8 C$ ). Three horizontal strips of label, resulting from the 3 injections, are visible in the ventral portions of ICc in this case (Fig. 9, bottom). In owl 182, in which the ${ }^{3} \mathrm{H}$-proline injection was made at a site tuned to a low frequency $(2.1 \mathrm{kHz}$ ) (Fig. $8 D$ ), the label field (of which there is only one) is located dorsally (Fig. 9, top). These results are consistent with the tonotopicity of ICc reported by Knudsen and Konishi (1978) and by Wagner et al. (1986). Label fields typically extend anteriorly from the level just posterior to where the tectal commissure becomes confluent at the midline. The label fields (Fig. 9) resulting from the 4 injections illustrated in Figure 8, when combined, form a vertical strip. Injections into those parts of NL not involved by 

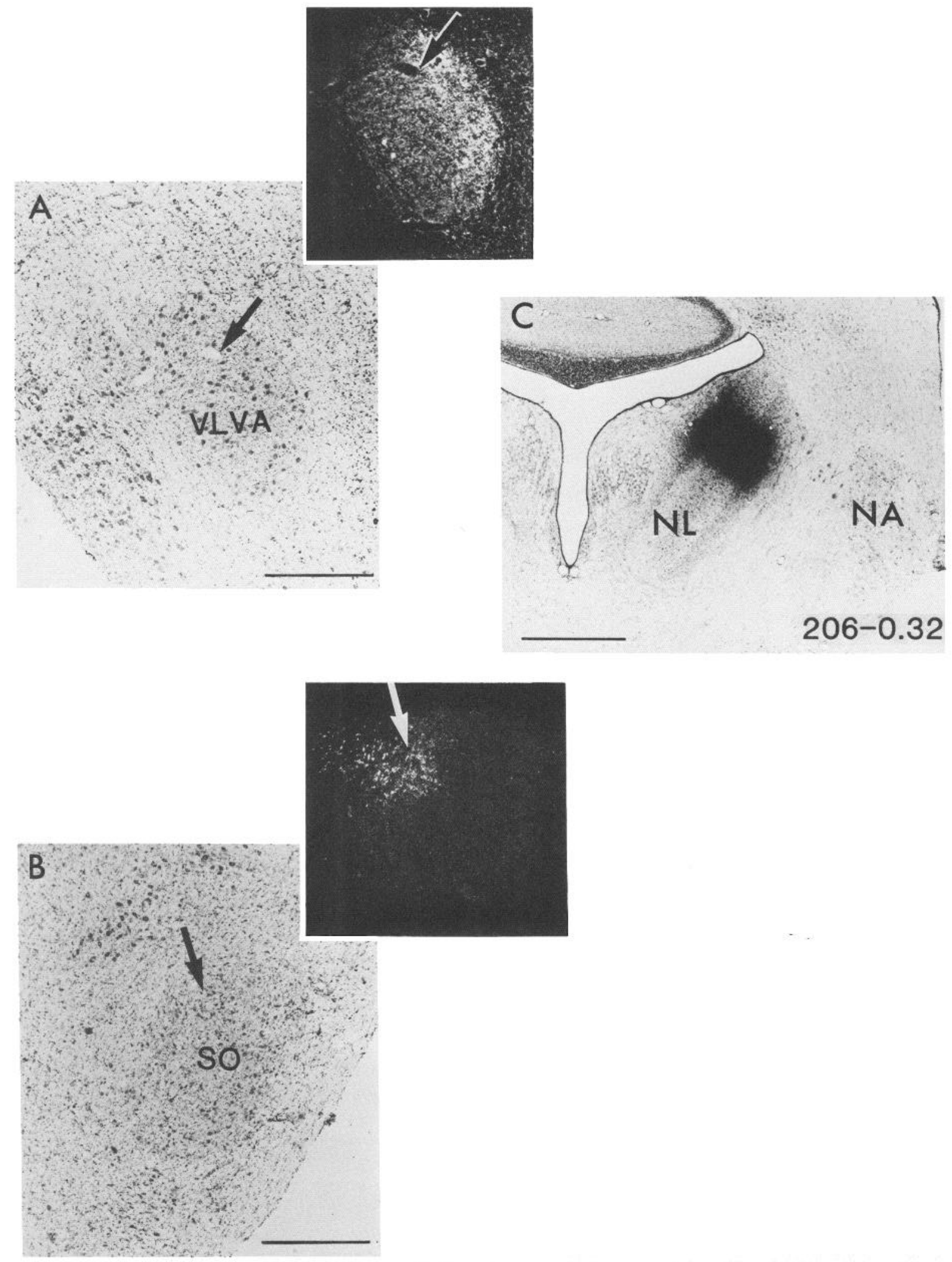

Figure 7. Autoradiographic label in VLVA and SO. Label in the contralateral (to the injection site) VLVA $(A)$ and ipsilateral SO $(B)$ following an injection of ${ }^{3} \mathrm{H}$-proline into nucleus laminaris $(N L)$ (owl 206 normalized ap level $0.32 \mathrm{in} \mathrm{NL}, C$ ) is shown in bright-field/dark-field pairs. The ipsilateral VLVA and the contralateral SO are unlabeled. The neurophonic response recorded at the injection site had a best frequency of $6.0 \mathrm{kHz}$. Scale bars: $A, B, 0.5 \mathrm{~mm} ; C, 1.0 \mathrm{~mm}$. 

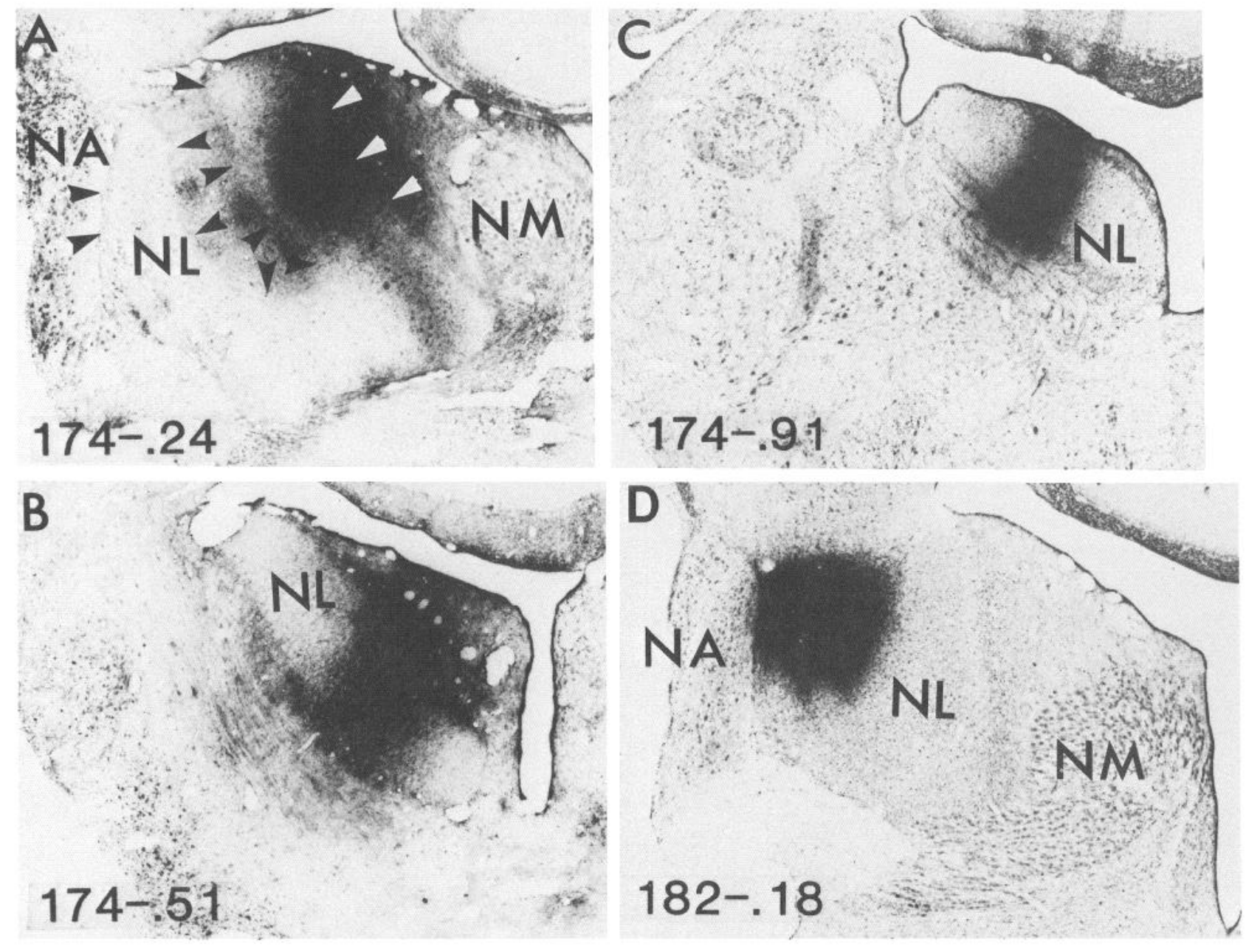

Figure 8. Four injection sites along the tonotopic axis of NL. Three of the 4 injections were made in one owl $(A-C)$, owl 174. The best frequencies of the neurophonic response at the injection sites were $5.1 \mathrm{kHz}(A$, normalized ap level, $0.24 \mathrm{in} \mathrm{NL}), 6.4 \mathrm{kHz}(B$, normalized ap level, 0.51$)$, and $7.4 \mathrm{kHz}(C$, normalized ap level, 0.91$)$. The fourth injection site, which is from another bird ( $D$, normalized ap level, 0.18$)$, owl 182 , elicited a neurophonic response tuned to $2.1 \mathrm{kHz}$. $A$, Borders of NL are marked by the tips of arrowheads. The injection sites in $A-C$ involve the fiber tract that contains axons of nucleus magnocellularis $(N M)$ but are otherwise confined to NL. The radiolabel in $D$ spreads to nucleus angularis $(N A)$, but this amount of contamination did not produce label in nuclei (i.e., contralateral VLVP, bilateral nucleus lemnisci laterale pars ventralis, and ipsilateral SO) that are diagnostic of the involvement of NA. Scale bar, which applies to all photographs, $1 \mathrm{~mm}$.

the 4 injections shown above produce no label outside the vertical strip. ICc ipsilateral to the injection is never labeled. The projections from NL to ICc, VLVA, and SO were confirmed with the retrograde transport of horseradish peroxidase (Takahashi and Konishi, 1985).

\section{Lesion experiments}

$\mathrm{CaBP}$ immunoreactivity has been reported in all somata of NL of the barn owl (Carr et al., 1985) and chicken (Jande et al., 1981). The congruence between the autoradiographic labeling pattern described above and the location of CaBP immunoreactivity thus suggests that the immunoreactive fiber plexus is composed of the terminals and distal portions of axons from NL. We tested this hypothesis by making an electrolytic lesion in a restricted portion of NL and treating the sections for $\mathrm{CaBP}$ immunohistochemistry. The results are shown in Figures 1012. At its center along the anteroposterior axis, the lesion consists of a hole in the lateral half of NL with a region of spongey tissue, devoid of somata, extending medially (delimited by the tips of arrowheads in Fig. 10). It occupies about one-third of the total nuclear volume and is centered, roughly, at a mid-anteroposterior level. A thin electrode track was observed in the cerebellum overlying NL. The neurophonic response, recorded at the lesion site before the current was passed, was tuned to $6.0 \mathrm{kHz}$.

The immunoreactivity in ICc and VLVA (Figs. 11 and 12, top right) of the right side, which is ipsilateral to the lesioned $\mathrm{NL}$, shows a normal staining pattern. On the left, which is the side innervated by the lesioned nucleus, the immunoreactive fiber plexus is absent from a restricted region of these nuclei (arrowheads, Figs. 11 and 12, top left). The gap in the staining in ICc and VLVA is found where a ${ }^{3} \mathrm{H}$-proline injection into the lesioned area of NL would be expected to produce label. No area that was consistently and convincingly devoid of the immunoreactive fiber plexus could be detected in SO of either side.

The gaps in the immunoreactive pattern in ICc and VLVA can be followed throughout ICc and VLVA and occupy similar areas on nearby sections, suggesting that the gaps are not an 

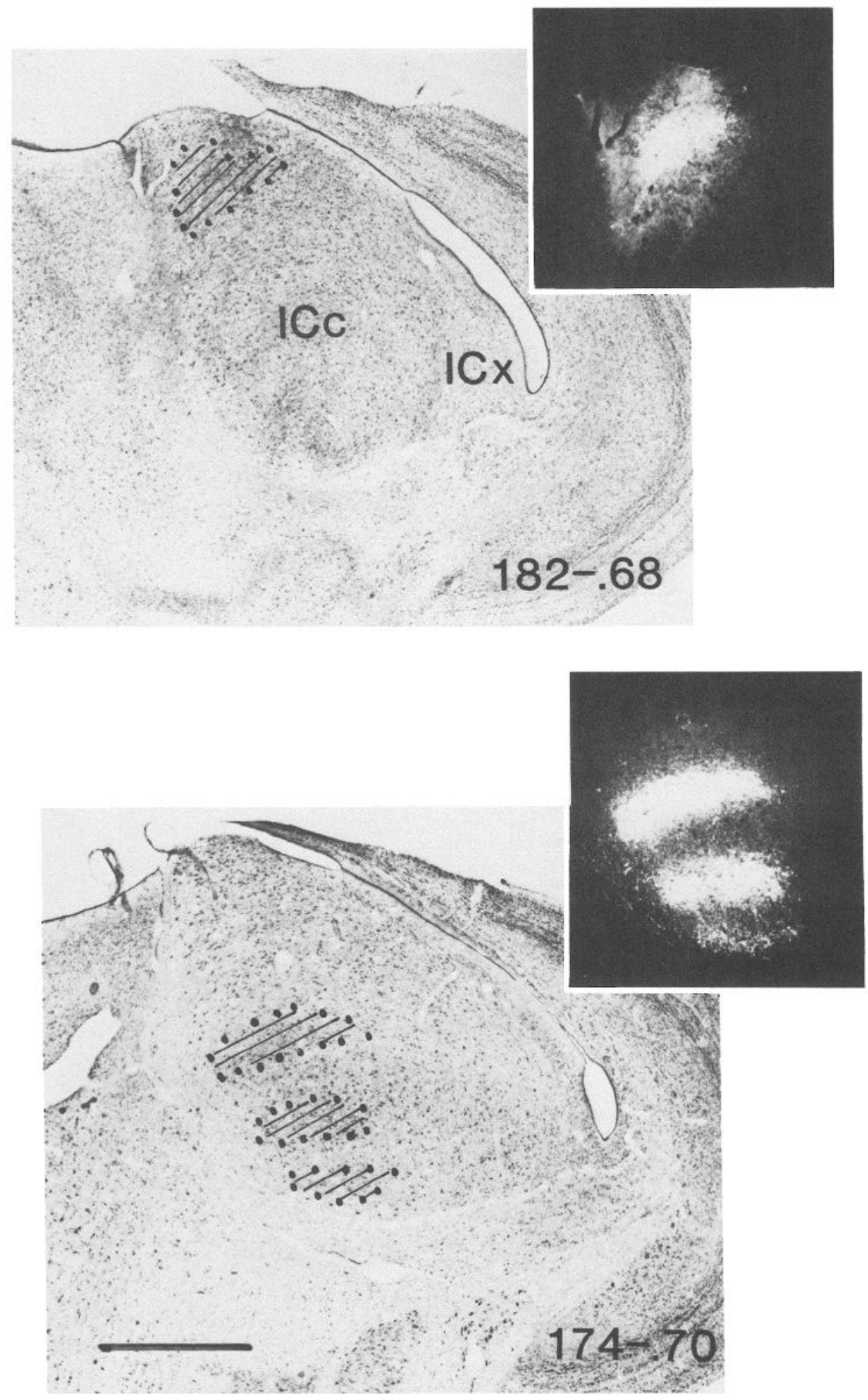

Figure 9. Label in the contralateral ICc produced by the injections shown in Figure 8. The site of the label shown in the dark-field photomicrograph has been drawn in on the bright-field photomicrograph. In owl 182 (top), one field of label is found dorsally within ICc. In owl 174 (bottom), which received 3 injections, 3 horizontal fields of label are visible in the more ventral aspects of ICc. Scale bar, which applies to all photos, $1 \mathrm{~mm}$. artifact of uneven staining. This evidence is supported by observation of alternate sections stained for degenerating fibers and terminals by the Fink-Heimer (1967) method (Figs. 11 and 12 , middle and bottom). On these sections, coarse argyrophilic fibers and granules form a zone in ICc and VLVA that complements the deficit in CaBP immunoreactivity. Thus, it may be seen in Figure 11 that the deficit in immunoreactivity and the zone of degeneration (delimited by the tips of arrowheads in the center left panel) occur at a roughly mid-dorsoventral level in ICc. Similarly, in Figure 12 the zone devoid of CaBPpositive fibers and the zone of degenerating fibers and terminals (arrowhead tips in the center left panel) are located at the medial edge of VLVA. In SO, sparse argyrophilic fibers and granules are scattered throughout the ipsilateral, but not the contralateral, nucleus.

Pathway-tracing studies in the pigeon (Leibler, 1975) and our 
Figure 10. Lesion in NL, demarcated by the tips of arrowheads, consists of a hole and, extending medially, a zone of spongey tissue, devoid of somata. The entire lesion occupies the mid-ap level of the nucleus and involves about onethird of the total volume of NL. Scale bar, $1 \mathrm{~mm}$.

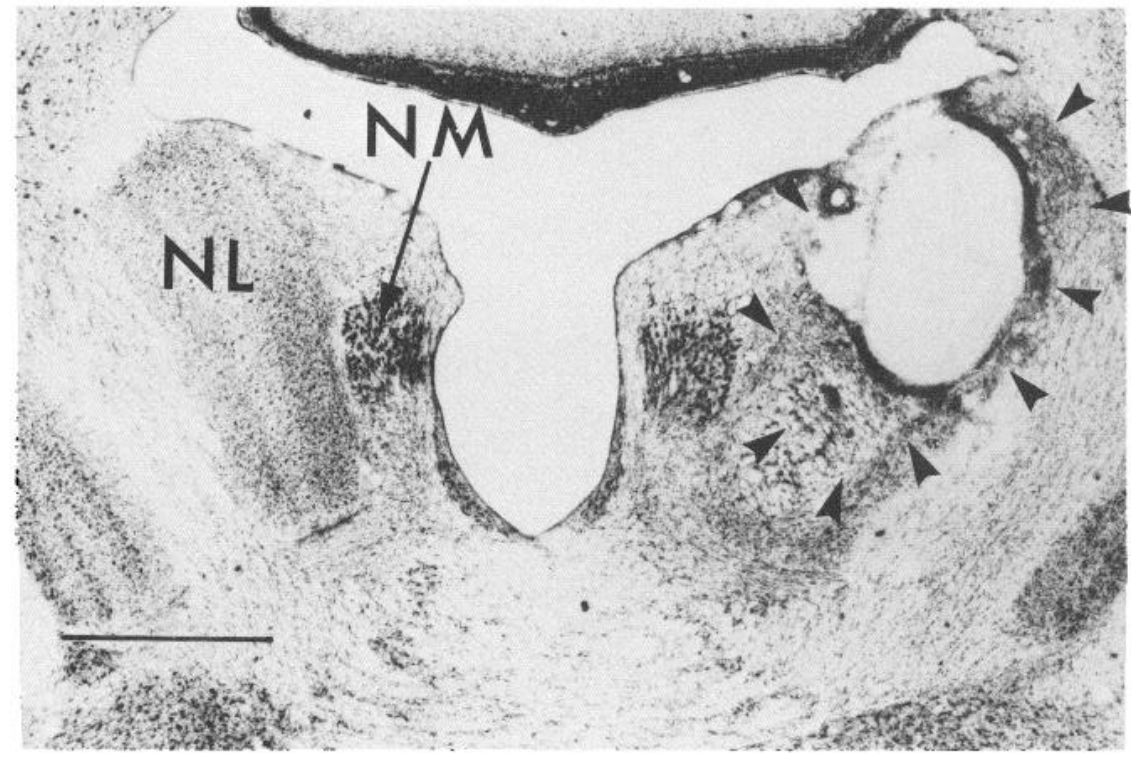

own studies in the owl suggest that the cerebellum overlying NL projects to neither ICc, VLVA, nor SO. Therefore, the degeneration observed in these nuclei could not be due to the destruction of cerebellar tissue by the passage of the electrode.

\section{Discussion}

The distribution of endogenous substances can potentially reveal much about brain organization, although the aspect of organization that is revealed is not always obvious. Cells can share a common chemical because the chemical is required for some aspect of signal processing. On the other hand, cells can share a common chemical marker simply because they share a similar developmental history. Functional organization is not necessarily revealed by studying the distribution of the latter marker. In order to be confident that the distribution of a chemical substance reveals an aspect of functional organization, the distribution of the substance should first be correlated with some element of connectivity or physiology. For example, cytochrome oxidase "blobs" of monkey striate cortex have been shown to be correlated with the presence of units that exhibit wavelength double-opponency and are not selective for orientation. Cells between blobs have the converse set of stimulus selectivities: They are orientation-selective and not double-opponent (Livingstone and Hubel, 1984). Cytochrome oxidase staining patterns are further elaborated in V2 of the monkey visual cortex, wherein DeYoe and Van Essen (1985) and Shipp and Zeki (1985) have shown that neurons in thick cytochrome oxidase stripes project to area MT. Neurons within thin stripes and regions intercalated between cytochrome oxidase stripes project, by contrast, to V4. Another example is AChE staining, which, in the caudate nucleus, is correlated with, and thus identifies, those zones innervated by the cortex (Ragsdale and Graybiel, 1981). In the above examples, a clear physiological or hodological association has been established for the staining pattern. Such histochemical markers are therefore useful for the operational definition of functionally relevant subdivisions that cannot otherwise be visualized in their totality without extensive surveys of connectivity and physiology. In the auditory system of the barn owl, NL is the site at which the interaural phase differences of the spectral components in sounds are encoded. CaBP im- munoreactivity stains the terminal field of NL and thus permits the operational definition of a major component of the circuitry devoted to the computation of interaural time difference.

We have presented evidence, above, that the distribution of the immunoreactive fibers and terminals is congruent with the terminal field of NL, as may be inferred by a comparison of immunoreactivity patterns and the distribution of radiolabel. In ICc and VLVA, furthermore, the immunoreactive fiber plexus disappears from a restricted part of each of these nuclei following a lesion that involves a restricted portion of NL. In $\mathrm{SO}$, tracing studies show that the terminal field of NL is sparse. This is consistent with the paucity of immunoreactive fibers in SO. It is also not surprising, therefore, that a clear-cut deficit in immunoreactivity could not be detected in SO following the lesioning of NL. We cannot exclude the possibility, however, that SO and, to a lesser extent, VLVA and ICc receive sparse, $\mathrm{CaBP}$-positive projections from another source in addition to NL.

The distribution of CaBP in the central nervous system has been studied in a number of vertebrate species. Lists of CaBPpositive structures, published for birds (Jande et al., 1981; Roth et al., 1981) and mammals (Jande et al., 1981; Feldman and Christakos, 1983; Garcia-Segura et al., 1984), attest to its widespread distribution. CaBP immunoreactivity in the avian auditory system has already been reported in that NM, NL, VLV, and inferior colliculus of the chicken are listed as containing immunoreactive fibers or cell bodies (Jande et al., 1981; Roth et al., 1981). These reports do not recognize subdivisions within VLV or IC that have been found in the pigeon, chicken, and owl (Leibler, 1975; Takahashi and Konishi, 1985, and unpublished observations; Conlee and Parks, 1986).

In the electrosensory system of Eigenmannia, a weakly electric fish, CaBP has been observed in all neurons that fire in synchrony, or "phase-lock," to the discharge of the electric organ (Maler et al., 1984). These include the pacemaker and relay neurons, which control the electric organ, as well as the spherical neurons of the electrosensory lateral line lobe and giant cells of the torus semicircularis, which sense and preserve the timing of electric organ discharge. In the torus semicircularis, which corresponds to the mammalian inferior colliculus, the axon end- 

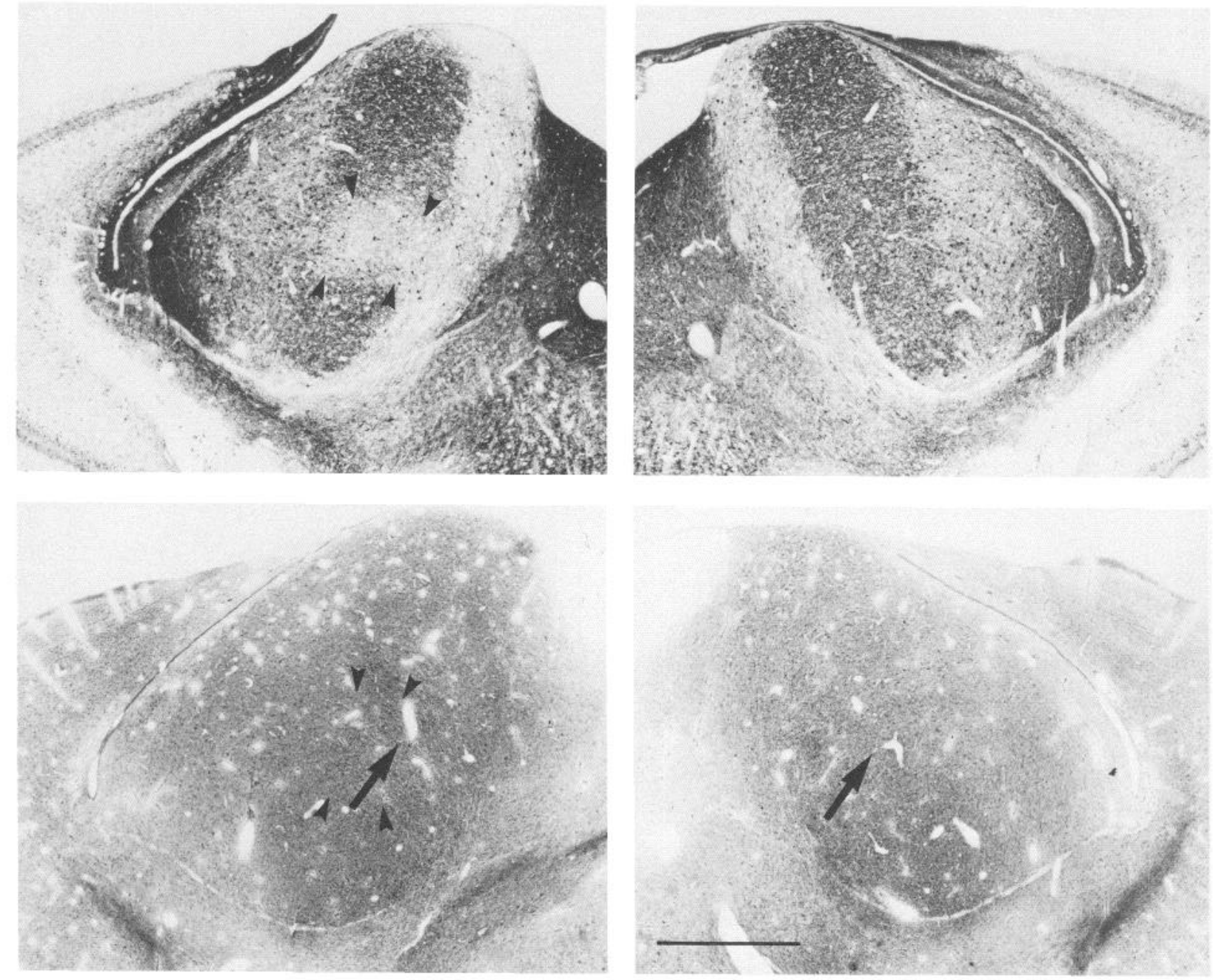

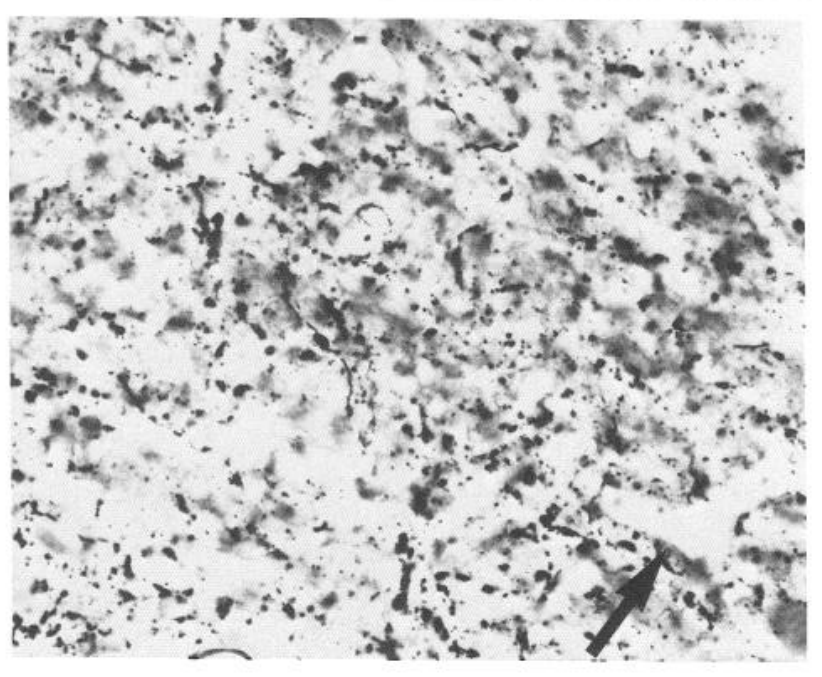

CONTRA.

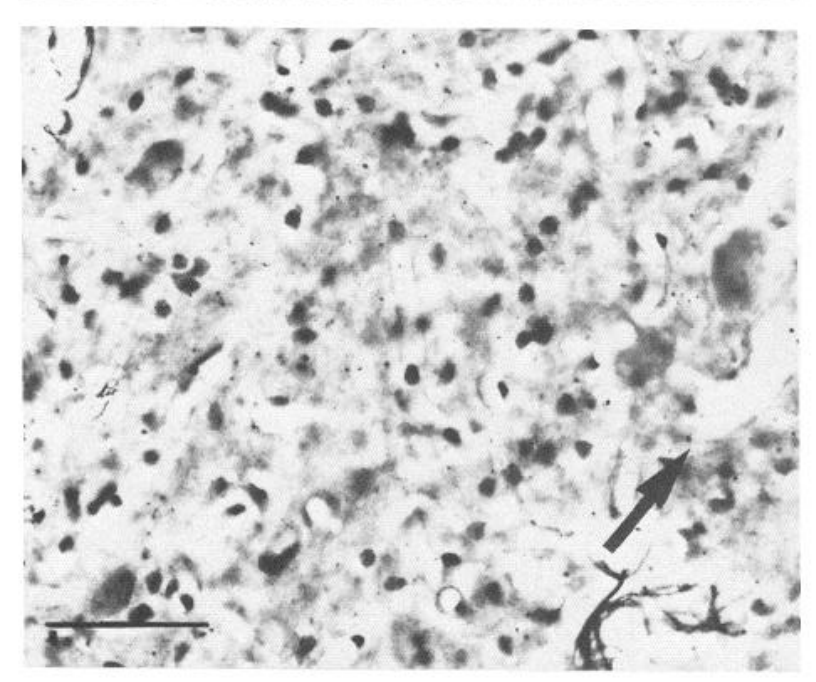

IPSI.

Figure 11. Staining in ICc after lesioning of NL. Top, Immunoreactive fiber plexus in ICc contralateral (left) and ipsilateral (right) to the lesioned NL. There is a gap (arrowheads) in the fiber plexus on the contralateral side, whereas the staining on the ipsilateral side is normal. Middle, Lowpower photomicrograph of a nearby $(60 \mu \mathrm{m}$ posterior) section processed by the Fink-Heimer method. On the contralateral side, a zone of argyrophilic fibers and granules (bottom left) is observed in the area corresponding to the deficit in staining (arrowheads, middle left). No such zone of degeneration is found on the ipsilateral side (middle and bottom right). Blood vessels that appear in the low- and high-power photographs (middle and bottom, respectively) are indicated by arrows. Scale bar in the middle right photo also applies to the top row of photos and represents $1 \mathrm{~mm}$. Scale bar in the bottom right photo also applies to the bottom left photo and represents $0.05 \mathrm{~mm}$. 

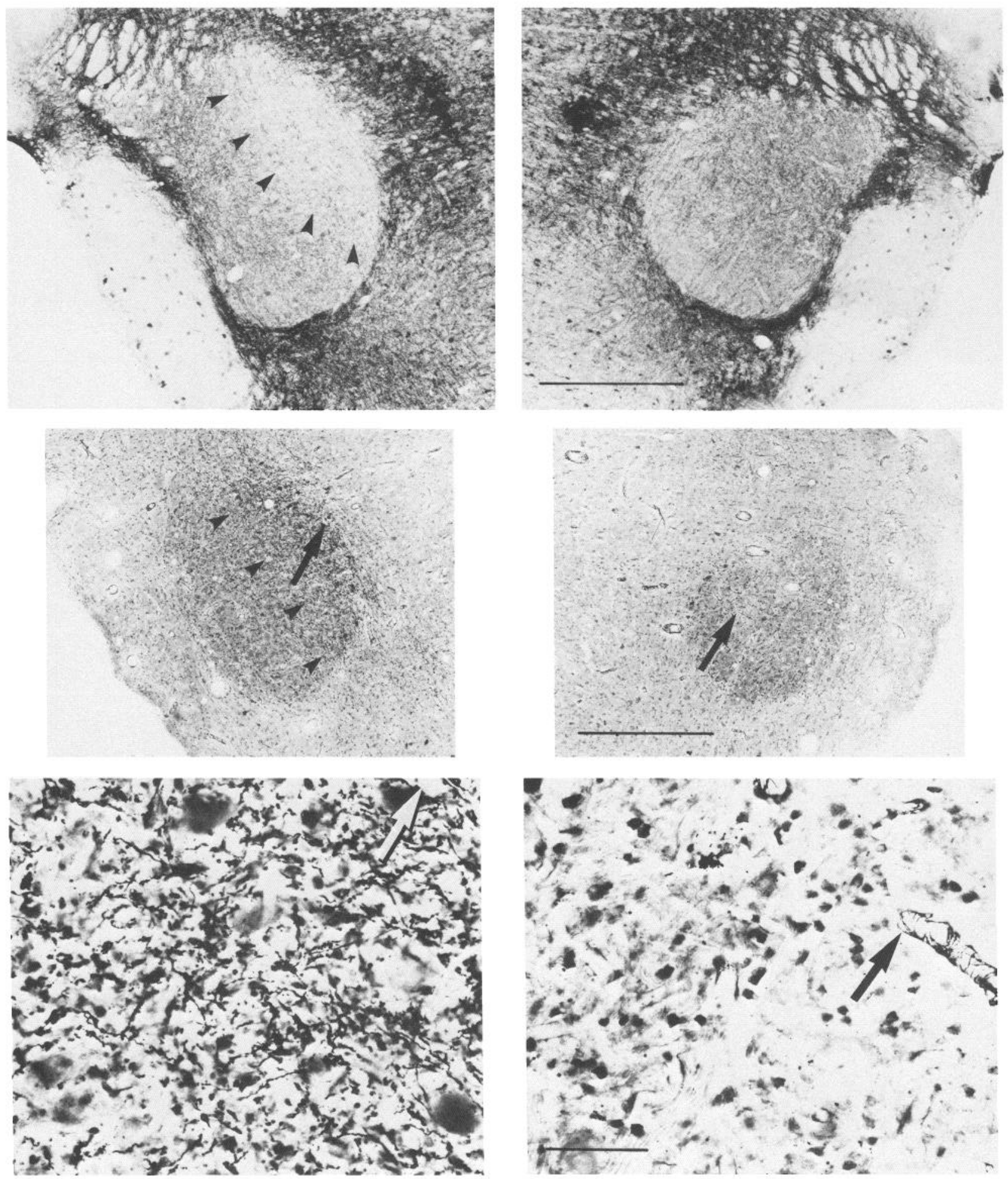

\section{CONTRA.}

IPSI.

Figure 12. Staining in VLVA after lesioning of NL. There is a deficit in the immunoreactive fiber plexus (top left, arrowheads) and a zone of argyrophilic fibers and granules in a corresponding spot of an adjacent section processed by the Fink-Heimer method (middle and bottom left). No such zones are observed on the ipsilateral side (right column). Scale bars in the top and middle right photos also apply to top and middle left photos and represent $1 \mathrm{~mm}$. Scale bar in the lower right photo also applies to the lower left photo and represents $0.05 \mathrm{~mm}$. 
ings of the giant cells form a CaBP-rich lamina. NM and NL, both of which have been reported to contain CaBP (Carr et al., 1985), are also composed of cells that faithfully replicate the temporal characteristics of acoustic signals (Sullivan and Konishi, 1984, 1986). It is therefore possible that CaBP is involved in mechanisms that underlie the preservation of timing of incoming signals in the postsynaptic somata.

Another role for $\mathrm{CaBP}$ has been recently suggested by Dye and Keller (1986), working in Apteronotus, another weakly electric fish. In this fish, sex hormones can cause changes in the frequency of its electric organ discharge. These authors report that implantation of dihydroxy vitamin $D$ causes a decrease in the frequency of electric organ discharge, the degree and time course of which are similar to those induced by implantation of estradiol. Noting that $\mathrm{CaBP}$ is found in the electroreceptive system of Apternotus, the authors speculate that the effect of vitamin $\mathrm{D}$ is mediated by $\mathrm{CaBP}$.

$\mathrm{CaBP}$ has also been associated with the tonic release of neurotransmitter (Sans et al., 1986). In the vestibular system of the cat, CaBP has been localized immunohistochemically in the Type II hair cells and in a subset of vestibular ganglion neurons (Sans et al., 1986). Type II hair cells, which are connected to afferent fibers having a high and regular spontaneous discharge rate, may tonically release neurotransmitter. Type I cells, which are only weakly immunoreactive, are connected with afferent fibers having irregular spontaneous activity (Sans et al., 1986). Neurons that tonically release transmitter may be found throughout the nervous system, and their distribution may explain the ubiquity of $\mathrm{CaBP}$.

There is no detailed study of CaBP distribution in the mammalian auditory system, although rosters of immunoreactive structures have been published. All neurons of the mammalian ventral cochlear nucleus, some of which phase-lock to acoustic stimuli (Lavine, 1971; Goldberg and Brownell, 1973; Rose et al., 1974), have been reported to contain CaBP (Jande et al., 1981; Garcia-Segura et al., 1984) in the rat. The medial superior olive, which also contains phase-locking neurons (Moushegian et al., 1967; Goldberg and Brown, 1969) and is thought to be the mammalian homolog of the avian NL (Ramon y Cajal, 1908 ), projects to a region in the ventrolateral portion of the ICc (Van Noort, 1969; Elverland, 1978; Henkel and Spangler, 1983). CaBP-immunoreactive fibers, which have been reported in the mammalian inferior colliculus (Garcia-Segura et al., 1984), may be restricted to the ventrolateral sector. Finally, it would be of interest to compare the distribution of CaBP immunoreactivity with the distribution of neurons of the mammalian inferior colliculus having various binaural properties (Roth et al., 1978; Semple and Aitkin, 1979; Wenstrup et al., 1986).

One study of the distribution of CaBP in the rat, by GarciaSegura and co-workers (1984), reports that somata of the medial superior olive of the rat are CaBP-negative. If this is indeed the case, the homology between the medial superior olive and the avian NL should be reexamined. It would also represent a counterexample to the association of CaBP with phase-locking ability. The study of Garcia-Segura et al. (1984), however, must be interpreted with caution. They define CaBP-negative somata as those that are counterstained with Evan's blue, but give no rcason why $\mathrm{CaBP}$ immunoreactivity, as visualized by their peroxidase method, should exclude the counterstain. It is therefore unclear how they were able to rule out the possibility that counterstaining masks light, but nonetheless positive, peroxidase reactivity.

\section{Appendix-List of Abbreviations}

ICc, central nucleus of the inferior colliculus; ICx, external nucleus of the inferior colliculus; LLV, nucleus lemnisci lateralis, pars ventralis; NA, nucleus angularis; NL, nucleus laminaris; NM, nucleus magnocellularis; OT, optic tectum; SO, superior olivary nucleus; TC, tectal commissure; VLVA, nucleus ventralis lemnisci lateralis, pars anterior; VLVP, nucleus ventralis lemnisci lateralis, pars posterior.

\section{References}

Carr, C. E., N. Brecha, and M. Konishi (1985) Organization of nucleus laminaris in the barn owl. Abstr. Soc. Neurosci. 11: 735.

Conlee, J. W., and T. N. Parks (1986) Origin of ascending auditory projections to the nucleus mesencephalicus lateralis pars dorsalis in the chicken. Brain Res. 367: 96-113.

Cowan, W. M., D. I. Gottlieb, A. E. Hendrickson, J. L. Price, and T. N. Woolsey (1972) An autoradiographic demonstration of axonal connections in the central nervous system. Brain Res. 37: 21-51.

DeYoe, E. A., and D. C. Van Essen (1985) Segregation of efferent connections and receptive field properties in visual area $\mathrm{V} 2$ of the macaque. Nature 317: 58-61.

Dye, J., and S. Keller (1986) A possible role for vitamin D of a sexually dimorphic neuronal oscillator in weakly electric fish. Soc. Neurosci. Abstr. 12: 834.

Elverland, H. H. (1978) Ascending and intrinsic projections of the superior olivary complex in the cat. Exp. Brain Res. 32: 117-134.

Feldman, S. C., and S. Christakos (1983) Vitamin D-dependent calcium-binding protein in rat brain: Biochemical and immunocytochemical characterization. Endocrinology 112: 290-302.

Fink, R. P., and L. Heimer (1967) Two methods for selective silver impregnation of degenerating axons and their synaptic endings in the central nervous system. Brain Res. 4: 369-374

Friedlander, E. J., and A. W. Norman (1977) A modified procedure for isolation of chick intestinal calcium binding protein. In Vitamin D: Biochemical, Chemical and Clinical Aspects Related to Calcium Metabolism, A. W. Norman, K. Schafer, J. W. Coburn, H. F. DeLuca, D. Fraser, H. G. Grigoleit, and D. V. Herrath, eds., p. 241, de Gruyter, Berlin.

Garcia-Segura, L. M., D. Baetens, J. Roth, A. W. Norman, and L. Orci (1984) Immunohistochemical mapping of calcium-binding protein immunoreactivity in the rat central nervous system. Brain Res. 296: $75-86$.

Goldberg, J. M., and P. Brown (1969) Response of binaural neurons in the dog superior olivary complex to dichotic tonal stimuli: Some physiological mechanisms of sound localization. J. Neurophysiol. 32: 613-636.

Goldberg, J. M., and W. E. Brownell (1973) Discharge characteristics of neurons in anteroventral and dorsal cochlear nuclei of the cat. Brain Res. 64: 35-54.

Henkel, C. K., and K. M. Spangler (1983) Organization of the efferent projections of the medial superior olivary nucleus in the cat as revealed by HRP and autoradiographic tracing methods. J. Comp. Neurol. 221: 416-428.

Jande, S. S., L. Maler, and D. E. M. Lawson (1981) Immunohistochemical mapping of vitamin D-dependent calcium-binding protein in brain. Nature 295: 765-767.

Karten, H. J., and W. Hodos (1967) A Stereotaxic Atlas of the Brain of the Pigeon, Johns Hopkins, Baltimore, MD.

Knudsen, E. I. (1983) Subdivisions of the inferior colliculus in the barn owl (Tyto alba). J. Comp. Neurol. 218: 174-186.

Knudsen, E. I., and M. Konishi (1978) Space and frequency are represented separately in auditory midbrain of the owl. J. Neurophysiol. 41: 870-884.

Knudsen, E. I., and M. Konishi (1979) Mechanisms of sound localization in the harn owl (Tyto alha). J. Comp. Physiol. 133: 13-21. 
I avine, R. A. (1971) Phase-locking in response of single neurons in cochlear nucleus of the cat to low frequency tonal stimuli. J. Neurophysiol. 34: 467-483.

Leibler, L. M. (1975) Monaural and binaural pathways in the ascending auditory system of the pigeon. Ph.D. thesis, Massachusetts Institute of Technology.

Livingstone, M. S., and D. H. Hubel (1984) Anatomy and physiology of a color system in the primate visual cortex. J. Neurosci. 4: 309356.

Maler, L., S. S. Jande, and D. E. M. Lawson (1984) Localization of vitamin D-dependent calcium binding protein in the electrosensory and electromotor system of high frequency gymnotid fish. Brain Res. 301: $166-170$.

Moiseff, A., and M. Konishi (1981) Neuronal and behavioral sensitivity to binaural time differences in the owl. J. Neurosci. 1:40-48.

Moiseff, A., and M. Konishi (1983) Binaural characteristics of units in the owl's brainstem auditory pathways: Precursors of restricted spatial receptive ficlds. J. Neurosci. 3: 2553-2562.

Moushegian, G., A. L. Rupert, and T. L. Langford (1967) Stimulus coding by medial superior olivary neurons. J. Neurophysiol. 30: 1239 1261 .

Pickel, V. M. (1981) Immunocytochemical methods. In Neuroanatomical Tract Tracing Methods, L. Heimer and M. J. Robards, eds., pp. 483-509, Plenum, New York, London.

Ragsdale, C. W., and A. M. Graybiel (1981) The fronto-striatal projection in the cat and monkey and its relationship to inhomogeneities established by acetylcholinesterase histochemistry. Brain Res. 208. 259-266.

Ramon y Cajal, S. (1908) Les ganglious terminaux du nerf acoustique des oiseaux. Trab. Lab. Invest. Biol. 6: 195-208.

Rose, J. E., L. M. Kitzes, M. M. Gibson, and J. E. Hind (1974) Observations on phasc-scnsitive neurons of anteroventral cochlear nucleus of the cat: Non-linearity of cochlear output. J. Neurophysiol. 37: 218-253.

Roth, G. L., L. M. Aitkin, R. A. Andersen, and M. M. Merzenich (1978) Some features of the spatial organization of the central nucleus of the inferior colliculus of the cat. J. Comp. Neurol. 182: 661-680.

Roth, J., D. Baetens, A. W. Norman, and L.-M. Garcia-Segura (1981) Specific neurons in chick central nervous system stain with an anti- body against chick intestinal vitamin D-dependent calcium-binding protein. Brain Res. 222: 452-457.

Sans, A., B. Etchecopar, A. Brehier, and M. Thomasset (1986) Immunocytochemical detection of vitamin D-dependent calcium-binding protein (CaBP-28K) in vestibular sensory hair cells and vestibular ganglion neurones of the cat. Brain Res. 364: 190-194.

Semple, M. N., and L. M. Aitkin (1979) Kepresentation of sound frequency and laterality by units in central nucleus of cat inferior colliculus. J. Neurophysiol. 42: 1626-1639.

Shipp, S. D., and S. Zeki (1985) Segregation of pathways leading from area $\mathrm{V} 2$ to areas $\mathrm{V} 4$ and $\mathrm{V} 5$ of macaque monkey visual cortex. Nature 315: 322-324.

Sullivan, W. E., and M. Konishi (1984) Segregation of stimulus phase and intensity coding in the cochlear nucleus of the barn owl. J. Neurosci. 4: 1787-1799.

Sullivan, W. E., and M. Konishi (1986) A map of interaural phase difference in the owl's brainstem. Proc. Natl. Acad. Sci. USA 83: 8400-8404.

Takahashi, T., and M. Konishi (1983) Functional organization of the barn owl's inferior colliculus. Soc. Neurosci. Abstr. 9: 212.

Takahashi, T., and M. Konishi (1985) Parallel pathways in the owl's brainstem auditory system. Anat. Rec. 211:191A.

Takahashi, T., and M. Konishi (1986) Selectivity for interaural time difference in the owl's midbrain. J. Neurosci. 6: 3413-3422.

Takahashi, T., A. Moiseff, and M. Konishi (1984) Time and intensity cues are processed independently in the auditory system of the owl. J. Neurosci. 4: 1781-1786.

Takahashi, T., C. E. Carr, N. Brecha, and M. Konishi (1986) An immunohistochemical marker for a functional pathway in the auditory system of the owl. Soc. Neurosci. Abstr. 12: 1276.

Van Noort, J. (1969) The Structure and Function of the Inferior Colliculus, Van Gorcum Assen, Netherlands.

Wagner, H., T. Takahashi, and M. Konishi (1986) The central nucleus of the inferior colliculus as an input stage to the map of auditory space in the barn owl. Abstr. Assoc. Res. Otolaryngol., p. 44.

Wenstrup, J. J., L. S. Ross, and G. D. Pollak (1986) Binaural response organization within a frequency-band representation of the inferior colliculus: Implications for sound localization. J. Neurosci. 6: $963-$ 973 